POS PROCEEDINGS

\title{
On improving composition measurements by combining compact Cherenkov telescopes with ground based detectors
}

\author{
Jan Auffenberg ${ }^{* 1}$, Thomas Bretz ${ }^{1}$, Martin Rongen ${ }^{1}$, Aatif Waza ${ }^{1}$, Christopher \\ Wiebusch ${ }^{1}$. \\ ${ }^{1}$ RWTH Aachen University, Institute for Particle Physics III A/B \\ E-mail: jauffenbaicecube.wisc.edu
}

\begin{abstract}
The cosmic ray knee is generally understood as marking the limiting energy of galactic accelerators and/or the onset of increasing outflow of particles from the galaxy. Detailed measurements of the cosmic ray composition are needed to support this prediction. The model and detector systematics of these measurements can be reduced with shower-by-shower based measurements employing independent detection techniques. Here we discuss the potential of compact imaging air Cherenkov telescopes to contribute to the composition measurement in interplay with ground based particle detectors.
\end{abstract}

35th International Cosmic Ray Conference

10-20 July, 2017

Bexco, Busan, Korea

\footnotetext{
*Speaker.
} 


\section{Introduction and motivation for a multi-component cosmic ray detector}

One of the central points of research around the high energy cosmic ray flux is improving the understanding of high energy particle physics. Besides the understanding of the general particle physics in an energy range beyond that reachable for todays accelerators, cosmic rays are also understood to be one of the keys towards the understanding of high energy particle acceleration and thus properties of the astronomical objects responsible for the cosmic ray production and/or acceleration.

High energy particle detection in astroparticle physics typically means the detection of very low fluxes of very bright events. To account for small fluxes in the order of events per year and $\mathrm{km}^{2}$ at the highest energies of the detected spectrum, large effective detection areas have to be realized. While direct detection methods require particle de-

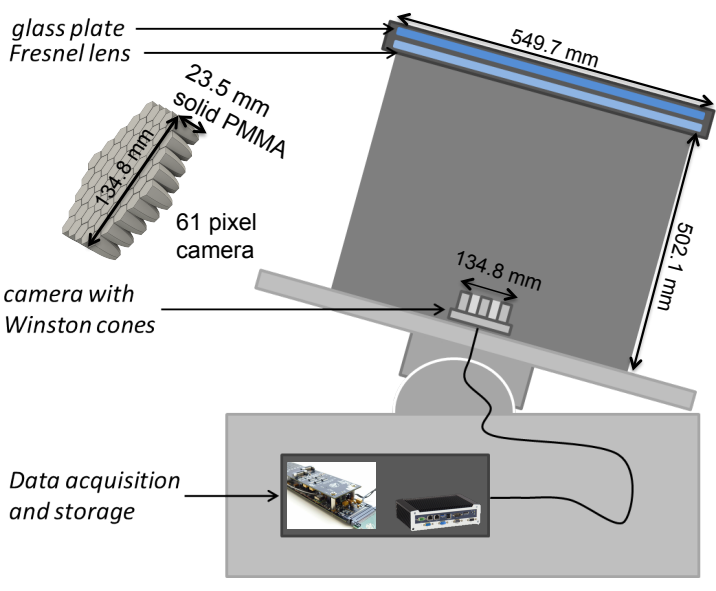

Figure 1: Drawing of the IceAct telescope prototype with a 61 pixel camera. The central part of the telescope is the camera with solid PMMA Winston cones and SIPMs for each pixel that detect the light collected by a $55 \mathrm{~cm}$ Fresnel lens. tectors within the cosmic ray induced airshower and particles directly hitting the detector, telescopes can detect light, emitted by particles far away from the detector. Emission mechanics are Cherenkov radiation of particles with energies around the speed of light and fluorescence. Both, the charged particle distribution of cosmic ray induced air showers on the ground and the light emission of charged particles in the air are key parameters to identify the properties of the primary particle. These proceedings discuss thoughts about adding imaging air Cherenkov telescopes to existing charged particle detectors for high energy comic rays on the example of the IceCube [1] and IceTop [2] detector. IceTop is located at the Geographic South Pole. It consists of 162 ice tanks of $\sim 2 \mathrm{~m}$ diameter that are equipped with two 10 inch PMTs each. The tanks are deployed over an area of about $1 \mathrm{~km}^{2}$ in about $125 \mathrm{~m}$ distance to each other directly above the deep IceCube detector. The size and the detector density yield IceTop's energy range from $400 \mathrm{TeV}$ to $200 \mathrm{PeV}$ [2]. The IceCube Neutrino Observatory deep in the ice is a one cubic kilometer large ice Cherenkov neutrino detector beneath IceTop, detecting mostly the Cherenkov light of down going muons from cosmic ray induced air showers. Due to the depth of the detector in the ice the energy threshold for muon bundles is in the order of several hundred TeV [1]. The combination of both detector components makes the IceCube Neutrino Observatory a unique detector to study the composition and energy of cosmic rays in the region of the knee. While spatial and directional resolution as well as the energy resolution are very good, the reconstruction of the cosmic ray primary particle depends on hadronic interaction models with remaining uncertainties [3]. Especially to constrain and resolve this uncertainties a third independent detection channel could be of great interest. An array of small imaging air Cherencov telescopes, 
refereed to as IceAct array [4], might provide this information.

\section{Criteria for an imaging air Cherenkov array at the South Pole}

In the following we briefly discuss the motivation and required properties of possible IceAct arrays that could be operated at the South Pole as a surface detector for cosmic-ray air showers in combination with IceCube and IceTop. Particle detector arrays on the ground like IceTop resolve only limited information about the particle evolution along the air shower axis. This is different for imaging air Cherenkov telescopes. As each telescope pixel of an IACT is monitoring the entire atmosphere in its field of view and the Cherenkov light emission is much more homogeneous compared to the distribution of the charged particles, we can directly detect height dependent information about the air showers.

In our case, the only requirement for IACTs is the sufficient detection of photons to be able to measure the shower development along the shower axis. Directional reconstruction and energy reconstruction are expected to be mainly driven by IceCube and IceTop. As a result the field of view of our pixels can be relative large above $1^{\circ}$ and the overall size of the telescope can be well below $1 \mathrm{~m}^{2}$. This drops the cost of a single telescope to well below \$ 10000 [5] including a DAQ that is based on Target7 [6] modules.

An IceAct array of the type described in [4] would add a powerful signal component for cosmic-ray physics with IceCube measuring the Cherenkov light emission of the electromagnetic component along the shower axis. This information is complementary to the high energy muon detection of the IceCube detector in the ice and the charged particle detection by IceTop on the ground.

\section{The current telescope design}

Efficiently running an Air Cherenkov telescope at the South Pole requires special treatment of the mechanics and electronics to account for the extreme environmental properties, including low temperatures and drifting snow but also the long periods of dark and clear weather [7]. This motivates to build a camera based on SIPMs [8]. Further design considerations follow from the planned use as a calibration device for IceCube and to veto and detect air showers [5]. Figure 1 shows a schematic of the current IceAct telescope design. In the following we describe the optical properties of the telescope along the signal path for incoming light. First, incoming light passes a 97\% UV-transparent glass plate (BOROFLOAT 33) that protects the entire following system from small snow crystals, dust and very cold temperatures. This is followed by a Fresnel lens with a $12^{\circ}$ field of view and a diameter of $549.7 \mathrm{~mm}$ (ORAFOL Fresnel Optics GmbH $510 \mathrm{~mm}$ SC 943 lens). A carbon cylinder sets the distance of the lens to the SiPM camera to $514 \mathrm{~mm}$ and ensures a shielding from scattered background light and snow. 61 specially designed PMMA Winston-Cones are sitting in front of the camera. They have a hexagonal entrance window and a quadratic output window focusing the light on $6 \times 6 \mathrm{~mm}^{2}$ SensL-J SIPMs (see Figure 2a). The radius $r_{f p}$ of the focal plane is $60 \mathrm{~mm}$, where the entrance window of the Winston cones is located in the focal plane of the Fresnel lens at $350 \mathrm{~nm}$ wavelength $(502.1 \mathrm{~mm})$. Figure 2 a summarizes these numbers. The overall light detection efficiency of the camera from the direction of $0^{\circ}-\delta$ is simulated to be in 
the order of $40 \%$ at $380 \mathrm{~nm}$ wavelength including the detection efficiency of the SensL-J $6 \times 6 \mathrm{~mm}^{2}$ SIPM and the limited fill factor for the new camera. In this study a camera design was used for the calculation that has a photon detection efficiency of $20 \%$ at $380 \mathrm{~nm}$ based on simulations.

Thus in this simulation, including the lens and the front glass the overall light detection efficiency of the telescope in its FOV is about $8 \%$ for Cerenkov light on the ground from 300-400 nm but will rapidly increase for future telescopes due the above mentioned camera design. Wavelength and direction dependent measurements are ongoing.

\section{Simulation of the light detection from cosmic rays of an IACT prototype telescope}

To get a first feeling on the potential of a small telescope to contribute to composition measurements together with IceTop a parametrized response of the telescope was developed. This was used to estimate the number of photons an IceAct telescope will detect for vertical cosmic ray induced air showers in $100 \mathrm{~m}$ distance to the shower core for different energies and primaries.

\subsection{IACT photon detection probability}

To correctly describe the efficiency of the telescope a GEANT4 [10] simulation of the telescope has been implemented to describe the light propagation through the telescope dependent on the hit point, direction and wavelength of the incoming photons [11]. To reduce simulation time, lookup tables where produced. They include the probability of a photon to get detected depending

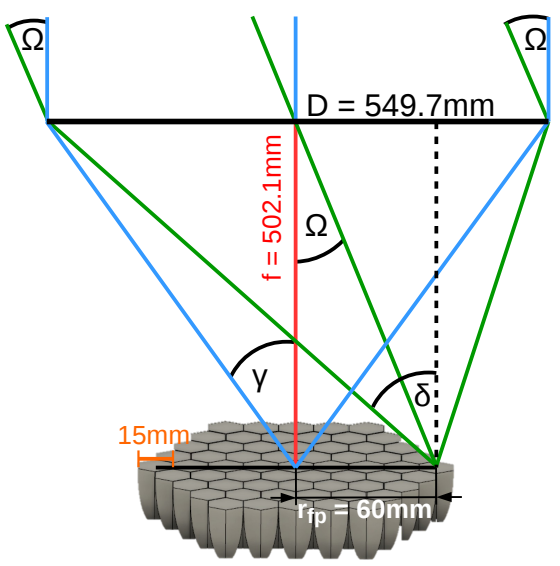

a) The acceptance angle that is required for the central Winston cone and a Winston cone on the side of the camera. $\gamma$ is $28.7^{\circ}$, $\delta$ is $33.7^{\circ}$.

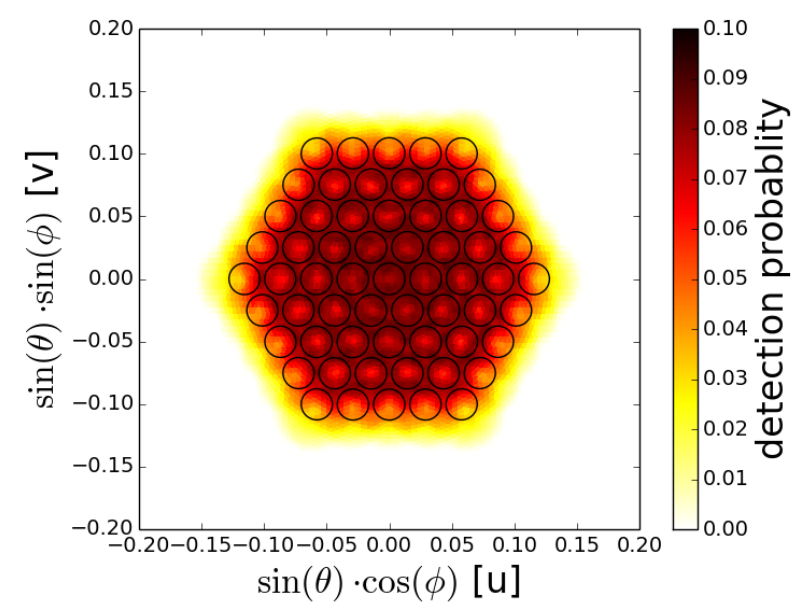

b) Detection efficiency for photons between $300-400 \mathrm{~nm}$ for one example IceAct telescope prototype dependent on the direction the photon hit the telescope lens in units of $u$ and $v$. The circles represent the positions of the Winston cone that collect light from certain direction.

Figure 2: The IceAct camera geometry and its photon detection probability determined by GEANT4 simulations. 


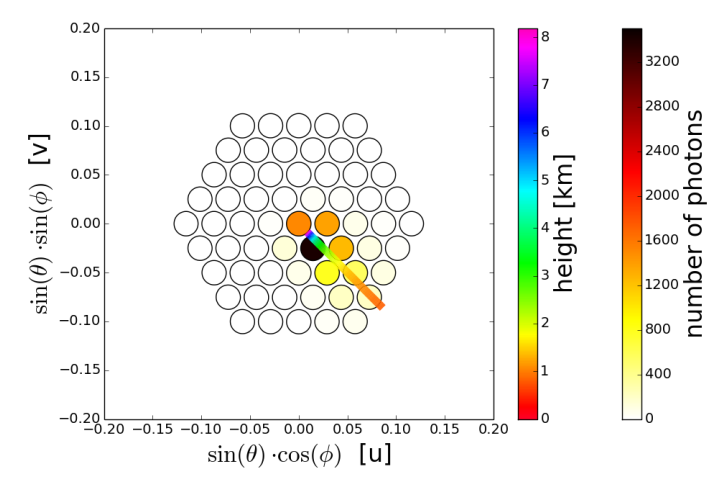

a) Simulated signal response of a vertical air-shower in an IceAct prototype telescope. Here the primary particle is an iron nucleus with an energy of $5 \mathrm{PeV}$.

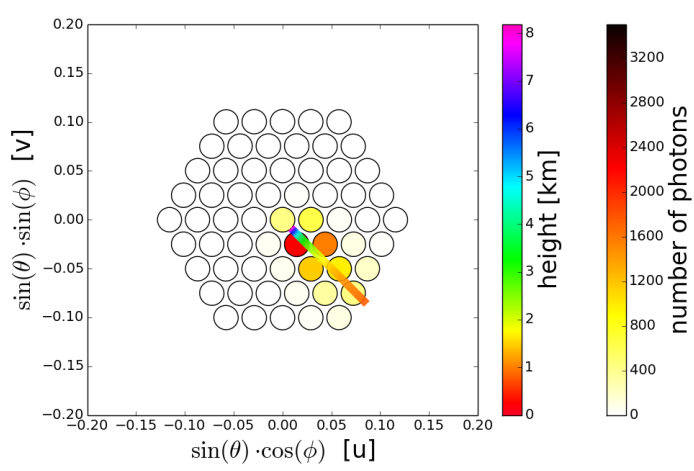

b) Simulated signal response of a vertical air-shower in an IceAct prototype telescope. Here the primary particle is a proton nucleus with an energy of $5 \mathrm{PeV}$.

Figure 3: The circles represent the positions of the Winston cone that collect light from certain direction in the plane of $\mathrm{u}$ and $\mathrm{v}$. The color-bar from white to black encodes the number of photons seen by each pixel. The rainbow color-bar indicates the value $h$, the projected height of the photons. The median of the height of all detected photons for this individual shower is indicated as a vertical line.

on the wavelength and the direction of a photon that hits the telescope entrance window (see figure 2b).

\subsection{IACT photon detection for air showers}

CORSIKA [12] simulations were used to simulate the amount of photons that reach the telescope for cosmic ray air showers. The parametrization shown in figure $2 b$ was used to estimate the number of photons we are going to detect in an IceAct telescope. As a first step only vertical showers in $100 \mathrm{~m}$ distance to the shower core were simulated. As primary particles iron and proton with $2 \mathrm{PeV}, 3 \mathrm{PeV}, 5 \mathrm{PeV}$, and $10 \mathrm{PeV}$ primary energy were chosen. Figure $3 \mathrm{a}$ shows the number of photons that were detected in the simulation for a single iron shower at $5 \mathrm{PeV}$ primary energy, vertically hitting the ground with the shower axis in $100 \mathrm{~m}$ distance to the telescope. The IceAct response simulated for figure $3 \mathrm{~b}$ the light detected from a proton shower with $5 \mathrm{PeV}$ primary energy and the same properties like the shower of Figure 3a. Note that IceTop can reconstruct the direction of air showers to the order of $1^{\circ}$ and the hit point on the ground can be reconstructed with an accuracy of about $25 \mathrm{~m}$ distance to the shower axis [2]. Thus we don't expect large changes in separation power from these parameters and don't discuss them in this proceedings, being aware that further studies will be needed.

Each pixel of the camera looks in one particular direction relative to the shower axis. To account for this fact the photon collection region of each pixel is translated into a projected height $h$ of the shower axis. The Figures $4 \mathrm{a}$ and $4 \mathrm{~b}$ show the distribution of $h$ for the two showers from figure $3 \mathrm{a}$ and $3 \mathrm{~b}$. 


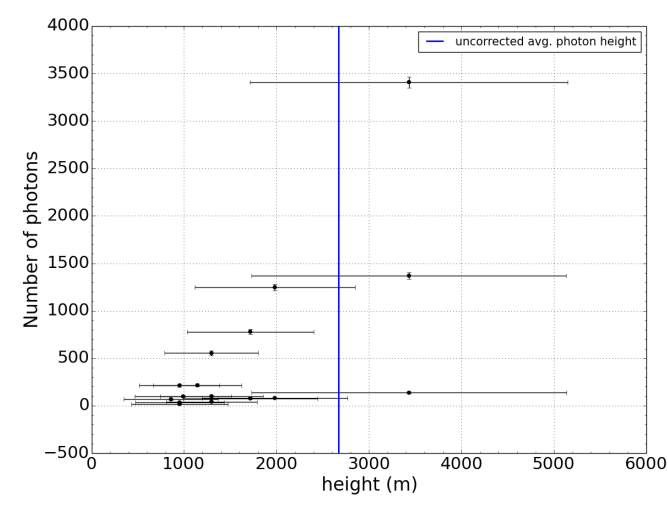

a) Simulated signal response of a vertical air-shower in an IceAct prototype telescope. Here the primary particle is an iron nucleus with an energy of $5 \mathrm{PeV}$.



b) Simulated signal response of a vertical air-shower in an IceAct prototype telescope. Here the primary particle is an proton nucleus with an energy of $5 \mathrm{PeV}$.

Figure 4: The figure shows the number of detected photons over the value $h$, the projected height of the photons, of each telescope pixel with signal photons. The error bars indicate the central region were $68 \%$ of the light in each cone was collected.

This can be used to calculate the median height of all detected photons for each individual shower. In the plots each median is indicated as vertical blue or red line. A distribution of these medians for 100 proton induced showers and 100 iron induced showers with $5 \mathrm{PeV}$ primary energy is shown in Figure 5a. The label 'corrected' on the x-axis indicates that the number of photons counted in each pixel on figure $3 \mathrm{a}$ and $3 \mathrm{~b}$ are weighted by their FOV width indicated by the error bars in the Figures $4 a$ and $4 b$. To measure the separation power of this distribution we choose the point of equivalence were the $\frac{\text { iron }}{\text { proton }}$ on the left side of the cut is equal to $\frac{\text { proton }}{\text { iron }}$ on the right side of the cut. This point of equivalence is shown as green line in Figure 5a on the left hand side. One can use this simple separation method for proton and iron showers at different Energies between $2 \mathrm{PeV}$ and $10 \mathrm{PeV}$. The separation power is found to be almost constant of the entire energy range (See Figure 5b).

Even if one uses a constant separation parameter of $h=2100 \mathrm{~m}$ the separation power only fluctuates between $88 \%$ and $98 \%$. This motivates that the separation of the primary particles is insensitive on uncertainties due to energy reconstruction.

\section{Summary and Outlook}

IceActs are small and robust telescopes that cost below $\$ 10000$. With being cost efficient they are a very good candidate technology to be deployed and operated in large numbers as cosmic ray detectors in harsh environments.

Only a small number of these telescopes measuring for example in coincidence with IceTop and IceCube might be sufficient to proof the principle of this idea.

We have described the idea of an enlarged IceAct array to improve the measurement of the cosmic ray composition together with IceCube and IceTop around the cosmic ray knee. First simulations 




a) Corresponding height $h$ distribution of 100 iron induced air showers with $5 \mathrm{PeV}$ primary energy and 100 proton induced air showers with $5 \mathrm{PeV}$ primary energy. The green line indicates the separation cut parameter $h$ that was chosen.

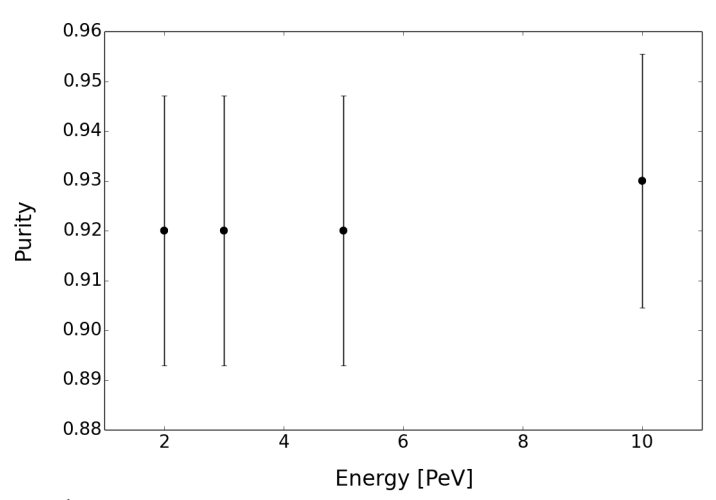

b) $\frac{\text { iron }}{\text { proton }}$ purity reached with the separation cut for primary energies from $2-10 \mathrm{PeV}$. This is equivalent $\frac{\text { proton }}{\text { iron }}$ ratio as we were choosing as the separation point the value were $\frac{\text { iron }}{\text { proton }}=\frac{\text { proton }}{\text { iron }}$. The label 'corrected' on the $\mathrm{x}$ axis means, that the total amount of photons measured in the pixels was corrected for its enlarged field of view.

Figure 5: Cosmic ray primary particle separation power on the example of proton and iron.

suggest a promising separation efficiency between proton and iron induced air showers at an energy range of $2 \mathrm{PeV}$ to $10 \mathrm{PeV}$.

The dependence of the separation power on uncertainties of the shower reconstruction parameters from IceTop, especially the direction and the hit point on the ground have to be carefully investigated.

This first test indicates that the $\frac{\text { Proton }}{\text { Iron }}$ separation seems to be stable and high over energies from $2 \mathrm{PeV}$ to $10 \mathrm{PeV}$. Refined simulation and analysis methods will have to show the real potential of IceAct telescopes to measure the composition of CRs in combination with IceTop and IceCube or other charged particle detectors on the ground.

Measurements of IceAct prototype telescopes in coincidence with existing air shower arrays will help to investigate the potential of such telescopes for cosmic ray detection and primary particle identification.

\section{References}

[1] A. Achterberg et al., The IceCube Neutrino Observatory: Instrumentation and Online Systems, JINST, 12 (2017), P03012.

[2] R. Abbasi et al., IceTop: A surface component for IceCube, Nucl. Inst. Meth. A, 700 (2013) 188-220, doi: 10.1016/j.nima.2012.10.067.

[3] IceCube Coll., Sensitivity of IceCube Cosmic-Ray measurements to the hadronic interaction models, PoS(ICRC2017) 319 these proceedings.

[4] IceCube-Gen2 Coll. IceAct: Imaging Air Cherenkov Telescopes with SiPMs at the South Pole for IceCube-Gen $2 \mathrm{PoS}(\mathrm{ICRC} 2017) 1055$ these proceedings. 
[5] J. Auffenberg et al., Study of air-Cherenkov telescopes for harsh environments like the South Pole with efficient air-shower detection below $100 \mathrm{TeV}$., PoS(ICRC2015)1047, https://arxiv.org/pdf/1511.01680.

[6] The CTA consortium, TARGET: toward a solution for the readout electronics of the Cherenkov Telescope Array, PoS(ICRC2015)932, https://arXiv:1508.06296.

[7] S. Benzvi et. al., An Estimate of the Live Time of Optical Measurements of Air Showers at the South Pole, PoS ICRC2015 (2016) 568, C15-07-30 proceedings.

[8] H. Anderhub et al., Design and Operation of FACT - The First G-APD Cherenkov Telescope, JINST 8 (2013) P06008.

[9] IceCube Coll., Sensitivity and Performance optimization of IceCube-Gen2, PoS(ICRC2017)GE06 these proceedings.

[10] S. Agostinelli et al., Geant4 - A Simulation Toolkit, Nuclear Instruments and Methods A 506 (2003) 250-303.

[11] T. Bretz et. al., FAMOUS - A fluorescence telescope using SiPMs, PoS ICRC2015 (2016) 649, C15-07-30 proceedings.

[12] D. Heck et al., CORSIKA: A Monte Carlo Code to Simulate Extensive Air Showers, Wissenschaftliche Berichte FZKA 6019 (1998). 